Commentary

\title{
Effects of Ionizing Radiation
}

\section{Kumar Alan Prem*}

Assistant Professor, Department of Biological and Environmental Sciences, Biomedical Institute for Regenerative Research (BIRR), Texas AËM University, Commerce, Texas, USA

Ionizing radiation (ionizing radiation) comprises of subatomic particles or electromagnetic waves that have adequate vitality to ionize iotas or atoms by segregating electrons from them. The particles by and large travel at a speed that's more noteworthy than $1 \%$ of that of light, and the electromagnetic waves are on the highenergy parcel of the electromagnetic spectrum. Gamma beams, $\mathrm{X}$-rays and the higher bright portion of the electromagnetic range are ionizing radiation, though the lower vitality bright, obvious light, about all sorts of laser light, infrared, microwaves, and radio waves are non-ionizing radiation. The boundary between ionizing and non-ionizing radiation within the bright range isn't strongly characterized, since distinctive atoms and molecules ionize at diverse energies, but is between 10 electronvolts $(\mathrm{eV})$ and $33 \mathrm{eV}$.

Ordinary ionizing subatomic particles incorporate alpha particles, beta particles and neutrons. These are regularly made due to radioactive rot, and nearly all are enthusiastic sufficient to be ionizing. Auxiliary infinite particles delivered after infinite beams connected with Earth's climate, and incorporate muons, mesons, and positrons. Infinite beams may moreover deliver radioisotopes on Soil (for illustration, carbon-14), which in turn rot and radiate ionizing radiation. Enormous beams and the rot of radioactive isotopes are the essential sources of common ionizing radiation on Soil, contributing to foundation radiation. Ionizing radiation is additionally produced falsely by X-ray tubes, molecule quickening agents, and nuclear fission. Ionizing radiation isn't perceptible by human faculties, so rebellious such as Geiger counters must be utilized to identify and degree it. Be that as it may, exceptionally tall power can deliver unmistakable light, such as in Cherenkov radiation. Ionizing radiation is utilized in a wide assortment of areas.

\section{Directly ionizing radiation}

Ionizing radiation is utilized in a wide assortment of areas such as medication, atomic control, inquire about, and mechanical fabricating, but presents a wellbeing danger on the off chance that legitimate measures against over the top introduction are not taken. Introduction to ionizing radiation causes cell harm to living tissue. In tall intense measurements, it'll result in radiation burns and radiation affliction, and lower level dosages over a extended time can cause cancer. The Universal Commission on Radiological Security (ICRP) issues direction on ionizing radiation security, and the impacts of dosage take-up on human wellbeing. Any charged molecule with mass can ionize molecules specifically by crucial interaction through the Coulomb drive on the off chance that it carries adequate motor vitality. Such particles incorporate nuclear cores, electrons, muons, charged pions, protons, and enthusiastic charged cores stripped of their electrons. When moving at relativistic speeds (close the speed of light, c) these particles have sufficient motor vitality to be ionizing capacity.

\section{Alpha particles}

These comprise of two protons and two neutrons bound together into a molecule indistinguishable to a helium core. Alpha molecule emanations are by and large created within the prepare of alpha decay. Alpha particles are a unequivocally ionizing shape of radiation, but when transmitted by radioactive rot they have moo entrance control and can be absorbed by a number of centimeters of discuss, or by the beat layer of human skin. More capable alpha particles from ternary parting are three times as lively, and enter proportionately more distant in discuss. The helium cores that frame $10-12 \%$ of infinite beams, are too ordinarily of much higher vitality than those delivered by radioactive rot and posture protecting issues in space. Be that as it may, this type of radiation is altogether ingested by the Earth's climate, which could be a radiation shield identical to approximately 10 meters of water.

\section{Beta particles}

Beta particles are high-energy, high-speed electrons or positrons transmitted by certain sorts of radioactive cores, such as potassium-40. The generation of beta particles is named beta rot. They are assigned by the Greek letter beta $(\beta)$. There are two shapes of beta rot, $\beta-$ and $\beta+$, which separately allow rise to the electron and the positron. Beta particles are less entering than gamma radiation, but more entering than alpha particles. High-energy beta particles may create X-rays known as bremsstrahlung ("braking radiation") or auxiliary electrons (delta beam) as they pass through matter. Both of these can cause an circuitous ionization impact. Bremsstrahlung is of concern when protecting beta emitters, as the interaction of beta particles with the protecting fabric produces Bremsstrahlung. This impact is more prominent with fabric of tall nuclear numbers, so fabric with moo nuclear numbers is utilized for beta source protecting.

${ }^{*}$ Corresponding to: Kumar Alan Prem, Assistant Professor, Department of Biological and Environmental Sciences, Biomedical Institute for Regenerative Research (BIRR), Texas A\&M University, Commerce, Texas, USA, E-mail: phcapk@nus.edu.sg

Received: July 09, 2021; Accepted: July 14, 2021; Published: July 20, 2021

Citation: Prem KA (2021) Effects of Ionizing Radiation. Bio Med 13: 439.

Copyright: 2021 (C) Prem KA. This is an open access article distributed under the terms of the Creative Commons Attribution License, which permits unrestricted use, distribution, and reproduction in any medium, provided the original work is properly cited. 